\title{
Structure Mechanism of Ordinary Matter Mass Formation
}

\author{
Sergey N. Golubev \\ Laboratory of Quantum Standards, All-Russian Research Institute of Metrological Service, Moscow, Russia \\ Email: sgolubev@vniims.ru
}

Received 22 February 2016; accepted 16 May 2016; published 19 May 2016

Copyright () 2016 by author and Scientific Research Publishing Inc.

This work is licensed under the Creative Commons Attribution International License (CC BY). http://creativecommons.org/licenses/by/4.0/

(c) (1) Open Access

\section{Abstract}

The famous paradoxes of quantum mechanics are created by the fact that elementary particles exist as the alternation between two structural states with different properties. This leads to probabilistic behavior, uncertainty principle, quantum tunneling, etc. The alternation of states plays the role of the frequency generator or clock. But for the objective character of quantum interactions the length standard also should exist in nature. Such analog of the rule must be physically real and in direct sense have to participate in the of particles interactions. Just this is the main role of physical vacuum. For such role vacuum should have quasi-crystalline geometry structure. Its symmetry requires in standard views only one fundamental change. In the quasicrystalline structure of the vacuum, the virtual shells of the real particles and atomic nuclei are not diffuse "clouds", as is assumed today. Virtual environments are clearly structured and rigidly quantised shells with the geometric structure similar to fullerenes. Such shells are forming for greater than $99 \%$ of the known substance mass. Virtual particles forming such shells belong to the group of bosons and probably are just Higgs bosons existing in the ordinary matter. Chemical fullerenes form a series of discrete geometric structures. In a similar manner virtual analogues of fullerenes form a series of discrete masses, which really exist in the nature as a set of elementary particles and atomic nuclei masses.

\section{Keywords}

Quantum Mechanics, Physical Vacuum, Quasi-Crystalline Structure, Mass Formation, Dark Matter, Higgs Bosons, Gravitation

\section{Introduction}

One of the central unsolved problems of all physics is the existence of a discrete set of masses of elementary 
particles and atomic nuclei, which somehow prove to be stable. In this article, we shall find the answer and even will be able to explain rations between the masses which really exist in nature. Why protons mass is 1836 more than electrons mass? Why there are no stable particles with intermediate values of the mass? About these issues thousands of researchers thought for decades. Below we shall calculate the ratio 1:1836 just in the mind, without any calculating devices.

We will try to understand the main principle of the structural organization of quantum particles in ordinary three-dimensional geometrical space and at a normal time, but not in different variants of abstract space which are used for traditional formal descriptions of quantum processes. The article offers new realistic interpretation of quantum mechanics and realistic explanation for mass formation of ordinary matter, actually the mass of atomic nuclei and elementary particles.

\section{Subject of Investigation}

According to standard theory around any atomic nuclei and any elementary particle exists something like the diffuse shell or the "cloud" of virtual particles. The main subject of this article is the true structure of these vacuum shells. The analysis shows that the shells have discrete and strictly quantum geometric structure which determines important properties of the particles. Such vacuum shells are absolutely necessary and very important structure components of atomic nuclei and elementary particles.

According to the standard theory the true value of the electron charge is not observable, and its tabular value is the result of attenuation due to the partial shielding by the vacuum. The consistency of the electron charge indirectly implies that vacuum shell has some stable structure. Below it is shown that the numerical value of the famous constant $137.03605 \ldots$ is created by vacuum shell with geometric structure similar to fullerene $\mathrm{C}_{60}$. A similar situation is also in the field of gravitation. Vacuum shells, composed of bosons and geometrically similar to the fullerenes, are creating more than $99 \%$ of the mass of atomic nuclei and elementary particles, i.e. the mass of ordinary matter. Structure and the nature role of these vacuum shells are the main subjects of this article.

\section{Methodology of the New Approach to Structure of Microcosm}

Particles with different masses have different structures. At the same time points do not have any internal structure by definition. Structural mechanism of mass creating does need to reject the view of particles as points. Today, such possibility has already appeared, but in the absence of methods for direct observations this requires a new approach to the problem.

The theory of relativity assumes that in each point of space there is a clock and the three-dimensional system of rules. In describing the macrocosm is quite enough to have the basic possibility to put a clock and a ruler to any desired point. In the formal description of the microcosm, this requirement is simply saved. In result we receive the look of a passive observer. For a participant in the events quantum measurement and interaction of particles can have an objective character only if in nature exists some physically real, not drawn on paper, analogue of clocks and rulers. Their presence seems to be impossible, but only at first glance. The operating principle of such clocks and rulers became clear after 40 years of electron microscopy research of the quantitative geometry of biological structures [1]-[8].

In result of such approach we see the world of elementary particles already not as a collection of paradoxes, but as a rational and elegant nature device. Nature occurs to be an excellent engineer, not a fan of mathematical puzzles. We change our view not only on elementary particles, but also on ourselves, primarily on the microscopic structure of our own body.

\section{Operation Principle of Natural Frequency Standard and Realistic Interpretation of Quantum Mechanics}

The natural frequency generator operates due to the fact that particles exist in the form of alternating two states with different properties. Just this alternation distinguishes elementary particles from small solids, as well as from atoms and molecules. This alternation of states creates the most famous paradoxes of quantum mechanics:

1) Probabilistic behavior. Quantum particles are involved in the physical interaction in both states but in completely different manners. Usually neighboring particles have random phase relation in alternation of states.

2) The uncertainty principle. The full range of particles properties is the sum of the properties of its two alter- 
nating forms. Because each particle moves the sum of properties can be determined only at such an interval of the particle movement when the both forms are present. This is the mathematical equivalent of the uncertainty principle standard definition.

3) The lack of classical trajectory. The line along which the particle moves in the form of spontaneous alternation of two states with different properties can be called the trajectory only conditionally. Such a line is not a classical trajectory. For this reason, the concept of classical trajectory does not apply to quantum particles.

4) The proper time of the particle. The period of oscillations with the alternation of states in terms of the Theory of Relativity corresponds to the concept of the proper time of the particle. At relativistic velocities, that quantum interconversion frequency decreases. At the speed of light, the alteration of states of photons and neutrinos disappears. The relativity theory calls this event the proper time stop of the particle.

5) Energy approach. Due to the alternation of states with different properties the force interactions between the particles attain a paradoxical specificity. That is why the energy approach is used for quantum particles instead force approach.

6) Geometry form and dimensions. The spontaneous attempts to represent the average view of two alternating states has led to the concept of particles without shape and size. Actually both alternating states of elementary particles have the size and geometric shape. At the first approximation, they are interconnected by a spatial inversion.

Four types of fundamental interactions for microcosm time scales do not appear simultaneously; instead, they belong to two tandems. Each tandem is peculiar to one of the two alternating states of a quantum particle, hence providing the main sense of the idea of the Great combination of physical interactions.

At one stage, the particle is involved in two types of interaction with a formally unlimited radius of action: electromagnetic and gravitational. At the transition to another state, the particle transforms into the proper diffraction picture. With this inversion of the geometric structure of the particle, its interactions with its theoretically unlimited radius of action transform into the interactions with a very small radius of action. Electromagnetic interaction proceeds to the weak nuclear interaction corresponding to the analytic theory of single electroweak interaction. Gravitational interactions with inversion of the structure of particles are converted into strong nuclear interactions.

According to standard theory, the electrostatic interaction between the charges is provided by the exchange of virtual photons, which are the quanta of alternating electromagnetic field. This aspect of the theory is fully consistent with our assertion of the existence of quantum particles in the form of the alternation of states with different properties. Specifically, at the scale of the microcosm, the electric charges and masses of the electron and proton are pulsating. At the stage of absence of electric charge the particles acquire the natural ability to tunnel through the electromagnetic barriers

\section{Operation Principle of Natural Length Standard, Proton-Electron Mass Ratio}

The alternation of states of quantum particles explains the paradox of their behavior. Peculiarities of their geometric structure and mass formation are explained by the operation principle of natural length standard. The role of such three-dimensional system of length standards or rulers plays the physical vacuum with the quasi-crystalline structure.

Ruler as the standard length requires at least two points. Therefore, a ruler cannot be inside each point of the microcosm. However, it is sufficient if each point forms part of the three-dimensional structure of natural rulers. It is impossible to obtain such rulers by returning to the classical hypothesis crystalline ether because all points of the classical ideal crystal cannot, in principle, be distinguished from each other. Therefore, the "division" of such rulers cannot, in principle, be digitised in any manner, either. The periodicity of the structure and the principal indistinguishability of its component points are, by definition, identical.

The necessary non-periodic structure was discovered by the famous British mathematician Roger Penrose approximately 50 years ago [9]. Nevertheless, for decades, three-dimensional Penrose patterns or pentagonal quasicrystals were perceived merely as entertaining mathematical puzzles. In 1984, quasicrystals were obtained in special metal alloys [10]. The Nobel Prize in Chemistry for obtaining metallic quasicrystals was awarded in 2011, approximately 30 years after the metal samples themselves and their diffraction patterns were obtained. Immediately following the discovery, the metal quasicrystals were considered "pseudoscience". Convincing evidence of the quasicrystalline of such ephemeral substance as the physical vacuum and such complex objects 
as living organisms require significant amounts of time to gather. The initial ideas of the quasicrystalline of the vacuum and biostructures were published in 1996 [3] [4].

The non-periodic filling of the volume, i.e., for quasicrystal construction, requires only two varieties of elementary cells, namely, two types of rhombohedrons of a special form for which the ratio of volumetric diagonals equals the famous proportions of the golden section. This requirement is neither mystical nor accidental but is the result of the appearance of quintic symmetry axes. The presence of the axes is incompatible with the periodicity of the structure and is prohibited for classical crystals. For aperiodic quasicrystal structures, however, such symmetry is inevitable. Quasicrystals do not violate the known theorem on the prohibition of crystallographic axes of symmetry of the fifth order, but go beyond its axioms. The proof of the theorem does not assume that co-existence of two types of elementary cells is possible.

A physical vacuum with an aperiodic quasicrystal structure can be compared with the gas of crystal dislocations and considered a type of synthesis of two classical hypotheses of crystalline and gaseous ether. Such a vacuum, however, does not contradict the Theory of Relativity; moreover it is required for its applicability to the microcosm, thereby ensuring the availability of an analogue line at each point. Features of the quasicrystal symmetry that do not allow using such a vacuum as an absolute reference system are discussed in detail in books [5] [6]. For half a century, the understanding of quasicrystals has transitioned from being a mathematical puzzle to corresponding to key structure of our world.

The transition from abstract geometry to the structure of the vacuum is associated with the peculiarities of symmetry. To implement the axis of symmetry of the fifth order it is necessary to have $5,10,15, \ldots$ points. To implement the plane of symmetry it is necessary to have $2,4,6,8, \ldots$ points. To implement the more complex combination consisting from the axis of symmetry of the fifth order and the plane of symmetry perpendicular to the axis it is necessary to have $10,20,30, \ldots$ points. To implement the entire set of symmetry elements of the quasi-crystall it is necessary to have $60,120,180, \ldots$ points. In other words, in the world with quasi-crystalline vacuum structure there are symmetry limitations for possible structures of virtual shells. Actually virtual shells of atomic nuclei and elementary particles are similar to fullerenes, of course only by the geometric structure. It must be clear that we are not talking about a hopeless attempt to extend the laws of chemistry on the atomic nucleus and the elementary particles, the similarity of the geometric structures is the result of the universality of the symmetry laws.

So, the condition for the existence of a natural standard of length is the quasicrystalline structure of the vacuum with infinitely large size. In the world based on such vacuum virtual shells of real particles and atomic nuclei acquire the structure of fullerenes. These structures have a certain finite size.

It is necessary to repeat and emphasize that for elementary particles we are talking about only one of the two alternating states. Geometric structure of another state of the same particle remains a subject for future research.

Today the foundations of the structure of fullerenes became widely known, so simple illustrations that I have presented in previous works, became redundant. But we will emphasize a number of features of these amazing chemical compounds of carbon that are important when comparing with the vacuum.

Today tools for industrial processing of diamonds are made from fullerenes. The high hardness of diamond was traditionally explained by the compactness of the atomic structure. The material from empty fullerene molecules occurs to be about $15 \%$ firmer. Such hardness and other unique properties of fullerenes are caused by the geometrical similarity of their molecules and the fundamental structures of the vacuum. In the unconscious form using of fullerenes in nanotechnology already started engineering using of physical vacuum effects.

Chemical fullerenes are formed only by carbon. It is the single element that creates chemical models of the vacuum shells, fractal increased in the size of course. The reason is associated with the numerical value of its mass and is explained in comments 2.2 to Table 2. The ability of living organisms to create analog models of vacuum structures goes much further. Despite the tremendous variety of organic substances, their carbon frame is displayed with high precision by a certain set of fragments, namely, only two crystal lattices of pure carbon: diamond and graphite. In any biological structure, such fragments of diamond and graphite lattices replace two types of rhombohedrons of a canonical quasicrystal, i.e., as a more complex quasicrystalline structure.

Direct geometrical similarity of the structures transforms biological cells in the analog models of physical vacuum world. Due to this biological cells live according to the laws of the quantum world without solving any equations.

Proton and electron mass ratio can be calculated simply in mind, without using any calculation equipment. Among all fullerenes the molecule $\mathrm{C}_{60}$ is distinctive. Elementary particles with a shell geometrically similar to 
$\mathrm{C}_{60}$ are the proton and the neutron. The algorithm for calculating the mass of these particles is more straightforward than expected. Real electric charge surrounded by the shell of 60 virtual particles becomes a complex of 61 particles.

Virtual particles do not have a proper weight, but the very existence of an ordered structure is possible only when specific interactions with each other and with the central core samples (i.e., with a real particle) occurs. In the system of 61 particles, only 1830 pairwise interactions are possible in a combinatorial sense. The contribution of each of them to the total mass of the proton equals the electron mass $m_{e}$ that is a natural standard unit of mass. The total contribution of the shell is $1830 \mathrm{~m}_{e}$.

The structure of the protons electric charge, the same is the structure of protons core, is not a subject of any chemical analogy but the subject of the quark theory. There are three quarks and three gluons which provide all possible combinations of interactions between quarks. Each quark mass and the dynamic equivalent of each gluon mass equal the electron mass $m_{e}$. Gluons have no resting mass; their mass is a relativistic mass equivalent. The overall proton core mass is $6 m_{e}$ and forms as the sum of the masses of three quarks with a mass $m_{e}$ of each and that of the three gluons, each weighing $m_{e}$ as well. The increase in the total mass of the proton to $1836 m_{e}$ is provided by a discrete virtual shell of 60 virtual particles.

The full estimated mass of a proton is $1836 m_{e}$, with the actual weight being $1836.15 \ldots\left(m_{e}\right)$, with $0.008 \%$ of the calculating error. The virtual shell of the proton and neutron are identical, but for dynamic reasons, the mass of the neutron core is $8.7 \mathrm{~m}_{e}$.

Protons shell consists of 60 virtual particles which are principally indistinguishable from each other. They belong to the group of bosons and perhaps can be identified with Higgs bosons. For the details of the comparison see below.

\section{Atomic Nuclei Mass Formation}

Empirically found algorithm for calculating the proton mass (in units of $m_{e}$ ) in general is universal. But for composite atomic nuclei it is necessary to take into account some features. Alpha particle mass and the mass of all other atomic nuclei are formed in the process, which gives a realistic view of the nature of the strong nuclear interactions. At the confluence of the protons and neutrons in the single atomic nucleus their individual shells of 60 virtual particles are lost. Instead them arises a single common shell for the entire atomic nucleus as a single entity. According to the standard theory of strong nuclear interactions are non-Central in nature. Just such forces should result from the formation of the common shell around atomic nucleus.

The shell of an alpha particle (helium nucleus) is similar to fullerene $\mathrm{C}_{120}$. Together with the core samples, they represent a set of 121 particles in which 7260 pairwise interactions are combinatorially possible. According to the empirically found algorithm they form a mass of $7260 m_{e}$. The core mass of the alpha particle is the sum of the masses of two protons cores and two neutron cores:

$$
(6+6+8.7+8.7) m_{e}=29.4 m_{e} .
$$

The full weight of the alpha particle is the sum of the core mass and the mass generating by the shell:

$$
(7260+29.4) m_{e}=7289.4 m_{e}=4.00 \mathrm{amu} \text {. }
$$

A similar mass calculation of chemical elements and their isotopes within the entire Periodic Table reveals the undeniable correlations between the structure of the atomic nuclei of the virtual shells and the real properties of the atoms themselves. The natural mechanism of mass formation is universal for all atoms of the Periodic Table of elements, as well as for the various elementary particles.

Algorithm used during protons and alpha particles mass calculating permits logical generalization. The result is an empirical equation for calculation atomic mass of all chemical elements. But the equation has important peculiarities. According to sense, the numbers of protons and neutrons, the numbers of virtual bosons in the shell are always only integer numbers. Integer equations are called Diophantus. But the name does not reflect the specifics of the equations, it reflects that physical meaning have only integer solutions of the equations. Such equations have two important features:

1) Such equations can be solved only by exhaustive search. Excluding very particular cases there are no general methods of solving.

2) Diophantine equations do not comply with the usual rule that one equation with two or three unknowns can- 
not be solved uniquely. Sometimes Diophantine equation with two or three unknowns has no solutions, and sometimes an integer solution is unique. Often there are several solutions, but one of them has obvious advantages in terms of symmetry or other parameters.

The calculation of the mass of the atomic nucleus using empirical Diophantine equations always includes modeling of geometric structures whose existence is demonstrated by all the independent data set. But for our purpose this is required. We are interesting not in the value of the atomic mass itself. We are interesting in the natural structure which forms just this mass value.

Algorithm of atomic mass calculation is reflected by empiric equation:

$$
A=\frac{m_{e}}{1822.8873}\left[\frac{n(n+1)}{2}+6 Z+8.7(A-Z)+Z\right]
$$

A-atomic mass in atomic mass units (amu);

$m_{e}$-electron mass;

$1822.8873 m_{e}=1 \mathrm{amu}$,- standard table coefficient, ratio between different mass measurement units;

$Z$-number of protons in nucleus and the number of electrons in atom;

$(A-Z)$-number of neutrons in nucleus;

(n) — number of virtual particles in nucleus virtual covers;

$\frac{n(n+1)}{2}$-number of all possible combinations of pairwise interactions in the system from $(n+1)$ particles,

it means from one real particle and " $n$ ” virtual particles;

$6 Z m_{e}$ - total mass (in $m_{e}$ units) of proton cores in nucleus, it means total mass of proton part in nucleus core;

8.7 $(A-Z) m_{e}$ - total mass (in $m_{e}$ units) of neutron cores in nucleus, it means total mass of neutron part in nucleus core;

$\mathrm{Zm}_{e}$ - total mass of electrons in atom, including of this member transform the calculation of nucleus mass to calculation of atomic mass - compare with usual tables of atomic and isotope masses became more useful. The influence on atomic mass of interactions between nucleus and atomic electrons is not counted.

Calculation results are given in Table 1 and Table 2. We emphasize again that the reality of bosonic shells confirms not by opportunity to present atomic weight as mathematical sum in Equation (3). Real existence of nucleus virtual shells is confirmed by correlations between shell structure and real properties of chemical elements, main correlations are mentioned in comments to the tables. With that said, we should keep in mind that the most interesting calculating parameter is not the value of atomic mass, but the number of bosons in the atomic nucleus shells, that is, the parameter (n) in Equation (3) and at the same time the leftmost column in the Table 1 and Table 2 .

Comments to Table 1.

1) Structure of virtual shells is based on pentagonal symmetry. Shell of 300 particles finishes the stage of fractal structure formation-appears 5 complexes with 60 particles in each. In the table for the first time appears the element with considerable weight part of not main isotopes.

2) Adding to the nucleus single neutron without any changing in shell structure increases calculated atomic mass only on neutron core mass which is $8.7 m_{e}=0.0048$ aum. Actual differences between isotope masses can be explained only by small changes in number of particles in virtual nucleus shell. Effectiveness of such mass corrections changes with atomic mass increasing along Periodic Table. Atomic mass increasing caused by addind a single virtual particle to nucleus shell is proportional to already existing number of particles. For berillium and silenium these numbers differences in three times-180 and 540 virtual particles correspondently. But the total nucleus mass is approximately proportional to the square of virtual particles number. That is why from berillium up to silenium atomic mass increases in 9 times. And in percent form the result of single particle adding to the nucleus virtual shell is approximately inverse proportional to the number of already existing particles. In the result $0.4 \%$ error of berillium atomic mass calculation is impossible to decrease by any correction of proposed number of particles in the shell_-adding of a single particle at once changes calculated mass for $1.1 \%$. For silenium adding of a single particle changes calculated mass only for $0.38 \%$, so decrising calculation error on $0.4 \%$ occurs to be trivial correction. Along Periodic Tables such corrections for proposed number of virtul particles in nucleus shells became efficient just at the same level at which in nature appears elements with considerable weight part of not main isotopes (about 10\%). 
Table 1. Masses of isotopes with nucleus shells with multiple to 60 numbers of virtual particles.

\begin{tabular}{|c|c|c|c|c|c|c|c|}
\hline $\begin{array}{l}\text { Number of virtual particles } \\
\text { in atomic nuclear shell }\end{array}$ & Element & Isotope & $\begin{array}{l}\text { Calculated } \\
\text { mass }\end{array}$ & $\begin{array}{l}\text { Actual } \\
\text { mass }\end{array}$ & $\begin{array}{l}\text { Calculated } \\
\text { error, \% }\end{array}$ & $\begin{array}{c}\text { Occurrence } \\
\text { of isotope, \% }\end{array}$ & $\begin{array}{c}\text { No. } \\
\text { of comment }\end{array}$ \\
\hline 60 & Proton & & $1836 m_{e}$ & $1836.15 m_{e}$ & 0.08 & & \\
\hline 120 & Alpha-particle & & 4.00 & 4.003 & 0.06 & & \\
\hline 180 & Beryllium & ${ }^{9} \mathrm{Be}$ & 8.98 & 9.01 & 0.3 & 100 & \\
\hline 240 & Oxygen & ${ }^{16} \mathrm{O}$ & 15.93 & 15.99 & 0.4 & dominate & \\
\hline 294 & & ${ }^{24} \mathrm{Mg}$ & 23.99 & 23.985 & 0.01 & 79 & 1.1 \\
\hline 300 & Magnium & ${ }^{25} \mathrm{Mg}$ & 24.88 & 24.986 & 0.4 & 10 & 1.2 \\
\hline 306 & & ${ }^{26} \mathrm{Mg}$ & 25.97 & 25.983 & 0.03 & 11 & \\
\hline 360 & & ${ }^{35} \mathrm{Cl}$ & 35.80 & 34.969 & 2.4 & 76 & 1.3 \\
\hline 360 & Chlorine & ${ }^{37} \mathrm{Cl}$ & 35.81 & 36.966 & 3.2 & 24 & \\
\hline 420 & Titanium & ${ }^{48} \mathrm{Ti}$ & 48.70 & 47.95 & 1.6 & 74 & 1.4 \\
\hline 480 & & ${ }^{63} \mathrm{Cu}$ & 63.60 & 62.940 & 1.0 & 69 & 1.5 \\
\hline 480 & Cooper & ${ }^{65} \mathrm{Cu}$ & 63.61 & 64.928 & 2.0 & 31 & \\
\hline 480 & Zinc & ${ }^{64} \mathrm{Zn}$ & 63.61 & 63.929 & 0.5 & 49 & 1.6 \\
\hline 540 & Selenium & ${ }^{80} \mathrm{Se}$ & 80.48 & 79.917 & 0.7 & 50 & \\
\hline 540 & & ${ }^{79} \mathrm{~B} \Gamma$ & 80.48 & 78.918 & 2.0 & 51 & \\
\hline 540 & Bromine & ${ }^{81} \mathrm{Br}$ & 80.48 & 80.916 & 0.5 & 49 & \\
\hline 600 & \multicolumn{6}{|c|}{ Neutral vector boson } & 1.7 \\
\hline 660 & Tin & ${ }^{120} \mathrm{Sn}$ & 120.19 & 119.902 & 0.24 & 33 & 1.8 \\
\hline 720 & Neodymium & ${ }^{143} \mathrm{Nd}$ & 143.01 & 142.91 & 0.07 & 12 & 1.9 \\
\hline 780 & Erbium & ${ }^{168} \mathrm{Er}$ & 167.83 & 167.932 & 0.06 & 27 & 1.10 \\
\hline 840 & \multicolumn{6}{|c|}{ Platinum and Iridium } & 1.11 \\
\hline 900 & \multicolumn{6}{|c|}{ Francium and Radon } & 1.12 \\
\hline 960 & \multicolumn{6}{|c|}{ Einsteinium } & 1.13 \\
\hline 1020 & \multicolumn{6}{|c|}{ Element yet without approved name predicated by me in 1996} & 1.14 \\
\hline 1080 & \multicolumn{6}{|c|}{ Element which in case it will be synthesized should have mass 321.3} & \\
\hline
\end{tabular}

3) For minimization calculation error up to $0.1 \%$ you want to assume that isotope ${ }^{37} \mathrm{Cl}$ have the nucleus shell of 366 particles, isotope ${ }^{35} \mathrm{Cl}$ - of 356 particles.

4) For minimization calculation error up to $0.1 \%$ you want to assume that isotope ${ }^{48} \mathrm{Ti}$ have the nucleus shell of 417 particles, calculated mass 48.01 .

5) For cooper isotopes middle mass is 63.546 . Calculation error relativly this value is only $0.085 \%$. Recall that middle mass of isotopes is always calculated with taking into account the relative occurance of isotopes. For minimization isotope masses calculation error you want to assume that isotope ${ }^{63} \mathrm{Cu}$ have the nucleus shell of 478 particles, isotope ${ }^{65} \mathrm{Cu} —$ of 485 particles. A similar reduction of error by fitting the estimated number of particles in the virtual shells further along the table, we will not comment.

6) Isotope ${ }^{64} \mathrm{Zn}$ with occurrence $49 \%$ is the main isotope of zink. Middle mass of zink isotops is 65.38 and deffers from mass of the main isotope more than one. Such great difference between middle mass and the main isotope mass occurs in the Periodic Table only few times. But Table 1 includes three such elements: zinc, selenium and tin.

7) Nuclei with shells of 600 virtual particles finish second stade of structure formation on the base of pentagonal symmetry. For nuclei with virtual shells of 300 particles calculated weight with the highest accuracy was in keeping with the magnesium isotope, relative frequency of occurrence of which is minimal. The trend continues on the nuclei with shells of 900 particles, such shells have not stable nucleuses of radon and francium. But just on the shells of 600 particles similar feature appears with maximum clarity.

Shell of 600 particles has an isotope of molybdenum ${ }^{99} \mathrm{Mo}$, actual mass 98.908 and calculated mass 99.34, calculation error $0.4 \%$. Half-life period is about 66 hours, this isotope is missing in nature. The same calculation 
mass and almost the same actual mass (98.906) has technetium isotope ${ }^{99} \mathrm{Tc}$. The half-life period is about 200,000 years. With minimal removal of mass from the "fatal" values of half-lives of technetium isotopes ${ }^{97} \mathrm{Tc}$ and ${ }^{98}$ Tc increase to approximately two and four million years, respectively. None of technetium isotopes almost never occurs in nature, although there are many isotopes in nature whose half-lives are considerably less than technetium. The lack of technetium is due to the fact that during natural nuclear reactions it is practically not formed and its natural resources are not renewed.

At first glance, the situation looks strange, because for reasons of symmetry in quasi-vacuum it is natural to expect that objects with virtual shells of 600 particles should be widespreaded. The main feature of these shells is that the main form of their existence in nature are not atomic nuclei but quite other particles-neutral vector bosons. Their mass is usually expressed in energy units; in fact it is almost identical with an atomic mass of technetium. Calculations of the mass of elementary particles, including neutral and charged vector bosons, see below. Vector bosons are the carriers of one of the four fundamental types of physical interaction-force weak nuclear interaction. In nature these are important and widespread particles, but because of the huge mass and short lifetime registration became technically possible relatively recently.

8) Isotope ${ }^{120} \mathrm{Sn}$ with occurrence $33 \%$ is the main tin isotope, see comment 1.6.

9) The most abundant neodymium isotope ${ }^{142} \mathrm{Nd}$ has occurrence $27 \%$. On reality neodymium do not have main isotope and there is no correlation between occurrences of isotopes and exactness of coincidence calculated and actual masses.

10) The most abundant erbium isotope ${ }^{166} \operatorname{Er}(34 \%)$ needs for correction of virtual particles number.

11) Platinum and iridium have accurate correlation between occurrences of isotopes and exactness of coincidence calculated and actual masses. For isotope ${ }^{195} \mathrm{Pt}(34 \%)$ calculation error is $0.2 \%$, for isotope ${ }^{194} \mathrm{Pt}(33 \%)$ calculation error is $0.3 \%$, for isotope ${ }^{196} \mathrm{Pt}$ (25\%) calculation error is $0.7 \%$, for isotope ${ }^{198} \mathrm{Pt}$ (7\%) calculation error is $1.7 \%$.

For iridium isotope ${ }^{193} \operatorname{Ir}(63 \%)$ calculation error is $0.9 \%$, for isotope ${ }^{191} \operatorname{Ir}$ (37\%) calculation error is $1.9 \%$.

12) Nuclei of francium and radon have virtual shells of 900 particles, calculated mass 223.40 in both cases. Francium have the single isotope ${ }^{223}$ Fr with actual mass 223.02, calculation error $0.17 \%$. For radon the most exactness of coincidence between calculated and actual masses have the most long-living isotope ${ }^{222} \mathrm{Rn}$, actual mass 222.02, calculation error $0.62 \%$.

Nuclei of francium and radon have virtual shells of 900 particles and they finish the third stage $(300,600,900)$ of fracture structure formation based on pentagonal symmetry. In addition to instability there are another two pecualiarities. Firstly after francium and radon in Table 1 there are only artifcial elements. And the second peculiarity is connected with values of atomic radius-see below.

13) Virtual shell of 960 particles has einsteinium nucleus with calculated mass 254.16. In entire group of artificial transuranium elements exactly einsteinium stands out by the presence of isotopes with a relatively high half-life-about a year. For isotop ${ }^{254}$ Es with a half-life of 275 days the error of mass calculation is only $0.03 \%$. The most long-lived isotope is ${ }^{252}$ Es with a half-life of 1.3 years. But the accuracy of the calculation of its mass is relatively small-error is $0.8 \%$. Reduction of error up to $0.01 \%$ requires reducing the expected number of particles up to 956 .

14) In the first publication of developing representations [4] I predicted the possibility of artificial synthesis of atoms with the shell of 1020 virtual particles and calculated mass 286.59. Then, in the calculation was neglected the difference between protons and neutrons masses, and also not included in the calculation the electrons mass. Consideration of these factors increases the calculated mass to 286.9. Predicted element has been synthesized in Dubna [11]. Its actual mass occurs to be about 288.

In the Periodic table the atomic radius varies with a distinct periodicity, which is complicated by a large number of smaller local minima and maxima. Almost all elements from the Table 1 are either directly at such minima points or tend to them. Directly in local minima are oxygen, chlorine, bromine, iridium, in the middle of two very shallow local minima are neodymium and erbium. For tin, the data are controversial, but poorly pronounced minimum, apparently, exists. On the edge of local minima are technetium, platinum, titanium, copper and zinc. For selenium the data are controversial and any interpretation is possible-selenium is the absolute champion of the Periodic table in the differences of atomic radius in measurements by different researchers.

The data on the atomic radius of radon is missing. Interpolation shows that the radius should be slightly larger than the xenon, and about the same as potassium. This severely disrupts the overall trend and is consistent with radon instability, the half-life of about 90 hours. Frances completely violates the General rule: he has the biggest 
radius of all the elements of the Periodic table. But since the half-life France is 22 minutes, it is the exception that just proves the rule.

From observed regularities, there is another exception of return character. There is a clear local minimum of the atomic radius, attributable to the iodine, which is not in the Table 1. But iodine also violates the General principle of the periodic table-it is lighter than the previous element, i.e., the atom falls out of the rule because it contains a lower number of neutrons.

In addition to diffraction measurements of atomic radii, there is a fundamentally different approach to estimate atomic sizes. The volume per one atom is calculated on the basis of the macroscopic density of the condensed phase and mass of one atom, expressed in macroscopic units. In the detailed reference there are such tables or graphics for at least part of the periodic table, but for independent calculation it is enough to have usual tables of density and atomic mass.

In this definition retains the overall relationship of the elements from Table 1 with local minima of the atomic radii, although some details of the picture change. In the evaluation of the atomic size on the basis of the macroscopic density there are no local minima per the halides—chlorine, bromine and iodine. But there is an additional minimum, which is magnesium. And beryllium together with boron and carbon, very little differing from each other, fall into the area of absolute minimum. In this definition of atomic volume they are the smallest atoms in the Periodic table. Not accidentally, the use of beryllium targets in many accelerator experiments. In this definition of atomic volume the hydrogen atom is much larger than atoms of beryllium or carbon. In my model this paradox has quite natural explanation: proton as the atomic nucleus of hydrogen preserves the alternation of two states with different properties and different sizes.

Fullerenes with multiple to 60 numbers of carbon atoms constitute only that part of fullerenes, which possess the highest symmetry. And chemical elements with multiple to 60 the numbers of particles in the virtual shells constitute only that part of the Periodic Table, which correspond to the minimum value of the atomic radii. Correlation between symmetry of the shell and geometric size is quite natural. Unexpected fact is the correlation with the size of the whole atom and not with the size of atomic nucleus. We must assume that the size of the shells close to the size of the entire atom and not to the size of its nucleus.

Experimental measurements of the atomic nucleus size refer to the core size. In experiments on the scattering even the existence of the virtual shells is not detected, therefore the measured sizes are not the sizes of the shell. The apparent contradiction of standard theory is poor compatibility with the official international table of atomic radii. For the most studied elements accuracy radii values is $0.01 \%$. It seems to be obvious that such accuracy is impossible for the radius of the diffuse blurred boundaries.

In the proposed model, the tabular values of atomic radii are the radii of discrete virtual shells, whose existence in the standard theory is not known. In other words, virtual shells remain unobserved for all available devices, but just the sizes of these shells actually are summarized in the existing today official international tables of atomic radii. The appearance of this paradoxical situation is explained in the book [5] separately for different types of chemical bonds (ionic, covalent and metallic).

Physical properties of the space inside the shell and outside are different from each other. The shell creates the main part of atomic mass of the atom. In terms of the theory of relativity the space inside the shell can be considered as curved. In terms of electromagnetism, one can assume that inside the shell and outside of it the dielectric constant of vacuum is different. These changes affect the nature of propagation of electromagnetic waves inside the shells. Firstly this applies to virtual photons, carrying out interaction between the nucleus and orbital electrons.

The specific laws of chemistry are the result of differences in the properties of the valence and inner electrons. In the proposed model this fundamental basis of all of chemistry is explained by the fact that the valence electrons are located outside the atomic virtual shell and the inner electrons are inside that shell.

The general picture is the following:

1) The size of the virtual shell is comparable to the size of the whole atom. More exactly, the shell separates the zone of completely filled electron orbits from the zone of valence electrons. The size of the virtual shell with high accuracy coincides with the size of the external completely filled electron orbit.

2) The highest sublevels, $d$ and f orbits, are always inside a virtual shell. That's why their electrons in chemical processes do not behave as valence electrons even in cases of partial filling of these orbits.

Founded algorithm atomic mass calculation applies in cases where the number of particles in the virtual shells is not multiple to 60 (Table 2). 
Table 2. Masses of isotopes with nucleus shells with not multiple to 60 numbers of virtual particles.

\begin{tabular}{|c|c|c|c|c|c|c|c|}
\hline $\begin{array}{l}\text { Number of virtual particles } \\
\text { in atomic nuclear shell }\end{array}$ & Element & Isotope & $\begin{array}{l}\text { Calculated } \\
\text { mass }\end{array}$ & Actual mass & $\begin{array}{l}\text { Calculated } \\
\text { error, \% }\end{array}$ & $\begin{array}{l}\text { Occurrence } \\
\text { of isotope, \% }\end{array}$ & $\begin{array}{c}\text { No. } \\
\text { of comment }\end{array}$ \\
\hline 85 & \multicolumn{2}{|l|}{ Deuterium } & $3670.7 m_{e}$ & $3671.3 m_{e}$ & 0.016 & Small & 2.1 \\
\hline 104 & \multicolumn{2}{|l|}{ Tritium } & 3.01 & 3.016 & 0.25 & 0 & \\
\hline 104 & Helium & ${ }^{3} \mathrm{He}$ & 3.01 & 3.016 & 0.28 & Small & \\
\hline 147 & \multirow[t]{2}{*}{ Lithium } & ${ }^{6} \mathrm{Li}$ & 5.99 & 6.015 & $0.4 \%$ & 7.5 & \\
\hline 159 & & ${ }^{7} \mathrm{Li}$ & 7.01 & 7.016 & 0.1 & 92.5 & \\
\hline 190 & \multirow{2}{*}{ Boron } & ${ }^{10} \mathrm{~B}$ & 10.00 & 10.013 & 0.2 & 20 & \\
\hline 199 & & ${ }^{11} \mathrm{~B}$ & 10.96 & 11.009 & 0.4 & 80 & \\
\hline 208 & Carbon & ${ }^{12} \mathrm{C}$ & 11.98 & 12.000 & 0.20 & Dominate & $2.2 ; 2.3 ; 2.4$ \\
\hline 225 & Nitrogen & ${ }^{14} \mathrm{~N}$ & 14.01 & 14.003 & 0.04 & Dominate & \\
\hline 262 & Fluorine & - & 19.04 & 18.998 & 0.24 & 100 & \\
\hline 269 & \multirow{2}{*}{ Neon } & ${ }^{20} \mathrm{Ne}$ & 20.01 & 19.992 & 0.1 & 90.5 & \\
\hline 282 & & ${ }^{22} \mathrm{Ne}$ & 21.99 & 21.991 & 0.03 & 9.2 & \\
\hline 288 & Natrium & - & 22.93 & 22.990 & 0.26 & 100 & \\
\hline 318 & Aluminium & - & 27.94 & 27.982 & 0.14 & 100 & \\
\hline 318 & Silicon & ${ }^{28} \mathrm{Si}$ & 27.95 & 27.977 & 0.1 & 92 & \multirow[t]{6}{*}{2.5} \\
\hline 335 & Phosphorus & - & 31.01 & 30.974 & 0.1 & 100 & \\
\hline 340 & Sulphur & ${ }^{32} \mathrm{~S}$ & 31.94 & 31.972 & 0.1 & 95 & \\
\hline 376 & Kalium & ${ }^{39} \mathrm{~K}$ & 39.04 & 38.964 & 0.2 & 93 & \\
\hline 380 & Argon & ${ }^{40} \mathrm{Ar}$ & 39.88 & 39.962 & 0.2 & Dominate & \\
\hline 380 & Calcium & ${ }^{40} \mathrm{Ca}$ & 39.87 & 39.963 & 0.2 & Dominate & \\
\hline 404 & Scandium & - & 45.07 & 44.956 & 0.26 & 100 & 2.6 \\
\hline$\ldots$ & $\ldots$ & $\ldots$ & $\ldots$ & $\ldots$ & $\ldots$ & $\ldots$ & 2.7 \\
\hline 450 & Iron & ${ }^{56} \mathrm{Fe}$ & 55.91 & 55.935 & 0.044 & 91 & 2.8 \\
\hline$\ldots$ & $\ldots$ & $\ldots$ & $\ldots$ & $\ldots$ & $\ldots$ & $\ldots$ & \\
\hline 923 & \multirow{2}{*}{ Uranium } & ${ }^{235} \mathrm{U}$ & 234.91 & 235.044 & 0.05 & 0.7 & \\
\hline 929 & & ${ }^{238} \mathrm{U}$ & 237.98 & 238.051 & 0.03 & Dominate & 2.9 \\
\hline
\end{tabular}

Comments to Table 2.

1) There exist so called endometallofulleren $M e C_{85}$. Each molecule is the complex of 85 carbon atoms in the shell and a single metal atom in the centre. Deuterium nucleus is the structure analogue of such molecule.

2) Nucleuses with proton or neutron numbers 2, 8, 20, 28, 50, 126, 208 have improved stability and are called "magic". Special status of these numbers or discrete structures also exist on the level of elementary particles shells. But among atomic nucleuses only carbon isotope ${ }^{12} \mathrm{C}$ has the shell of "magic" number of virtual particles-208 particles. Such shell has extremely interesting peculiarity. Isotope mass ${ }^{12} \mathrm{C}$ with accuracy of approximately $2 \%$ coincides with the mass difference between two particles carriers of one of the four types of fundamental interactions: weak nuclear forces. Such particles, as is widely agreed, are charged and neutral vector bosons.

Electromagnetic resonances arise when the photon energy matches the energy difference between two levels of an electron and sometimes other particles. Resonances in the physical vacuum arise at the interaction with mass (energy) equal to the mass difference between charged and neutral vector bosons. Greater than $99 \%$ of all known substances create the virtual shell of nuclei, whose geometric structure is similar to that of fullerenes. Carbon, forming the chemical fullerenes, is the only element that creates chemical analogues of the most fundamental structures of the physical vacuum. Of course, the molecular analogues are created with a fractal increase in the absolute size. This similarity of structures is the real cause of the extraordinary properties of chemical fullerenes.

These direct geometric similarities of the structure of matter and the structure of the physical vacuum are keys 
to many biological problems. The possibilities of living organisms in terms of the creation of such chemical imitations or analogue models of physical vacuum shows greater potential. The uniqueness of carbon is directly related to the existence of life. For a proper understanding, it should be added that the occasionally re-emerging hypothesis concerning the possibility of life based on silicon is a misunderstanding, regardless of recent results. Silicon does not form compounds with aromatic type of connections, and without the latter, it is impossible to obtain an analogue of biochemistry.

3) Atomic nucleus of the main carbon isotope $C^{12}$ has a shell of 208 virtual particles. A complex of 208 protons and neutrons constitutes the nucleus of lead $\mathrm{Pb}^{208}$. There is also a specific fractal, the existence of which cannot be displayed as part of the conceptual apparatus of the standard theory. Among the many toxic heavy metals, lead in this regard does not stand apart. However, this element has a unique ability to penetrate into the body through intact skin. Fractality by definition signifies the presence of a geometrical similarity amongst structures, in this case, subatomic structures. A general rule, i.e., "similar dissolves similar", has been known to the field of chemistry since the times of alchemists.

4) In tandem carbon—silver surprising effect can be seen. Silver has two isotopes $\mathrm{Ag}^{107} \mathrm{and}^{\mathrm{Ag}^{109}}$ with practically equal occurrences. For the formation of the conditionally averaged atomic mass of 108 units would be required shell of 624 virtual particles that is exactly 3 times more than 208 particles in the carbon shell. The atomic masses 108 and 12 units differ from each other exactly 9 times. Atomic volumes of silver and carbon are respectively 10.27 and 3.42 (diamond), i.e. differ from each other practically exactly 3 times.

In this case virtual particles in the shells of silver and carbon are identical by their characteristics. In all other cases, changes in the structure of nuclear shells lead to significant changes in the atomic mass, which corresponds to nuclear reactions and higher energies. And only between the silver and the carbon isoenergetical exchange of virtual particles in the composition of the shells became possible.

In addition to the specifics of the bactericidal properties of silver recall classic photo. Attempts to get a photo without silver failed. And attempts to substitute by other materials photographic gelatin (technically processed collagen protein) also failed. The photographic process is clearly based on the unique properties of tandem silver-carbon and is more complicated than it is common taken to think. In the standard interpretation of photographic developer is considered to be simply a chemical reducing agent of silver. But in all molecules of actually used developers there are aromatic rings. For using as reducing agents, they do not hinder, but do not help. Their constant presence in the structure of molecules developers does not correspond to a simple explanation of the photographic process.

5) Among the minerals of the earth's crust the weight part of aluminosilicates is more than the part of other minerals all together. This propensity of aluminum and silicon to the formation of joint compounds indirectly related to the identical structure of the virtual shells.

6) During the Periodic Table scandium firstly starts filling side of the subgroup. There is a real correlation with the fact that the scandium crosses important structural milestone of 400 particles in a virtual shell.

7) In Table 1 and Table 2 there are included the most important isotopes of all elements in the interval from hydrogen to scandium, without any lapses. After scandium calculation of the mass of all elements and isotopes becomes trivial fitting task (see note 1.3). In this part of periodic table I shell review only two interesting examples, the iron and uranium.

8) Iron in the periodic table corresponds to the energy optimum of nuclear forces. In this respect, it is remarkable small error in calculating the atomic mass of the main isotope of iron. The number of particles in the iron shell is exactly in the middle between the two "canonical" shells from 420 and 480 particles.

9) Transuranic elements that can be created artificially, finish periodic table on the level at which the number of virtual particles in the shell is equal to about 1000. It is just the limit for the ensemble of particles, which can be arranged according to geometric laws of clusters. This structural limitation have geometry reason and have universal application: chemical homogeneous clusters also can contain only about 1000 atoms. With further increase in the number of atoms arise notoriously heterogeneous system in the form of a mixture of microcrystals, clusters and fragments with random amorphous structure. Calculation of transuranic elements mass can be performed in the scope of virtual shells model (Table 1).

Adding to the vague end of transuranic part of the periodic table there is exactly clear undisputed border: natural part of the periodic table ends by isotope of uranium ${ }^{238} \mathrm{U}$. A unique feature of the nucleus ${ }^{238} \mathrm{U}$ is quite remarkable value for the mass of its shell. The nucleus consists of 92 protons and 146 neutrons. Its mass is the sum of weights 92 proton cores and 146 neutron cores. In units of $m_{e}$ the mass of the core ${ }^{238} \mathrm{U}$ is: 
$6 \times 92+8.7 \times 146=1822.2$. This is $99.25 \%$ of the total proton mass. Almost exact match with the full mass of the proton is not accidental. But even such accuracy estimate is understated, with respect to the discrete structure of the atomic nucleus, the accuracy is $100 \%$. Creation of the next element means adding to ${ }^{238} U$ nucleus one proton core and at least one neutron core. As a result, the boundary of the total proton mass must be crossed. But such nuclei are not formed as a result of natural processes. We will see below that the intersection of the boundary by core mass of vector bosons leads to the emergence of the phenomenon of "negative mass" (!). This structural restriction refers just the natural process of nuclei formation, but not to their existence. That is why artificial production of transuranic elements became possible.

\section{Masses of Elementary Particles}

The masses of elementary particles are calculated with the same algorithm. But unlike atomic nuclei, many elementary particles have a fractal system of multiple virtual shells. The main interest in this article is not the computation of particle mass itself, but empirical attempt to find such geometric structure of bosons shells, which is forming just really existing mass values. In all cases the criterion of the correction for the geometric reconstruction is a structural alignment with other properties of the same particles.

According to the standard theory the strong interactions have such property as saturation. In my model of vacuum shells this view is clarified by empirical fact that the energy of each pairwise interaction is strictly quantized, taking the following values:

1) In the shells with the number of virtual particles 60 and over this energy is $m_{e}$. This value is by five orders of magnitude more than the characteristic energy of one chemical covalent bond. At this energy there is a saturation of bonds between virtual particles in nuclear shells. Just because of this when calculating the mass of atomic nuclei we have not met with any attenuation coefficients for the intensity of the interactions.

2) In shells of less than 60 virtual particles energy equivalent of single interaction is smaller because interactions are not saturated.

And their energy is rigidly quantized and takes the values:

- In shells of 20 - 40 particles equal to $\left(m_{e} / \pi\right)$

- In shells of less than 20 particles equal to $\left(m_{e} / 4 \pi\right)$.

The appearance of the number $\pi$ as the coefficient of attenuation of interactions occurs also in the calculation of the electromagnetic constant $1 / 137$ when the accuracy of calculation is $0.0001 \%$ (see below). It is no doubt that the appearance of the number $\pi$ is caused by some exact structure reason. But for its understanding we need more detailed information.

\subsection{Mass of Heavy Leptons}

This group includes two types of particles that do not participate in strong nuclear interactions. Properties of heavy leptons are analogues of electrons or positrons, but differ from them multiple increased mass.

a) Hypothetical reconstruction gives the following structural picture. The mass of the Tau-lepton is created by

the real electron mass in the role of primary generator and fractal system of three virtual shells in the role of

gravitational amplification. Structure of three shells is the following:

1) The shell of 11 particles. Together with the real electric charge with mass $m_{e}$ (an obvious analogue of the electron) forms a complex of 12 particles in which 66 pairwise interactions are possible combinatorial. With such a small number of particles the contribution of each interaction to the total mass the Tau lepton is not $m_{e}$, but $m_{e} / 4 \pi$. The appearance of $\pi$ is the empirical fact, but we will meet it again several times. Together with the mas of the electric charge $\left(m_{e}\right)$ the contribution of this complex to the total mass of the Tau lepton is:

$$
m_{e}+(66 / 4 \pi) m_{e}=6.2521 m_{e} .
$$

It is quite obvious that in ordinary space complex of 12 particles has the shape of an icosahedron with 12 vertices.

2) The shell of 20 particles, the role of core performs the first shell. In a system of 21 particles 210 pairwise interactions are possible combinatorial, contribution of each interaction is $m_{e} / \pi$. The contribution of this shell to the total mass of the Tau lepton is

$$
(210 / \pi) m_{e}=66.8451 m_{e} .
$$


It is quite obvious that in ordinary space the shell from 20 particles has the shape of a dodecahedron with 20 vertices.

3) The shell of 82 particles, the number 82 is one of the so-called "magic" numbers. In the role of core performs the second cover together with the first shell inside her. In the complex of 83 particles 3403 pair interactions are possible combinatorial. The contribution of each pairwise interaction in a total mass equal to $m_{e}$ and coincides with the contribution of each pairwise interaction in the formation of the mass of the proton, and atomic nuclei. The whole contribution of the shell to the total mass of the Tau-lepton is $3403 \mathrm{~m}_{e}$. Within the meaning of the algorithm this value can be only integer.

Total calculated mass of the Tau-lepton is the sum of the three masses:

$$
6.2521 \cdot m_{e}+66.8451 \cdot m_{e}+3403 \cdot m_{e}=3476.0972 \cdot m_{e} .
$$

Actual mass is $(3477.5 \pm 6) m_{e}$. High accuracy of coincidence of the actual and estimated mass confirms the correctness of particles structural reconstruction.

Some comments about shell of 82 particles are necessary. Atomic nuclei with the number of protons or neutrons 2, 8, 20, 28, 50, 82, 128, 208 are the most stable. Actually with these numbers are connected structures of increased stability. Conventionally they are usually mentioned as "magic" numbers. It is natural to assume that similar structures exist at the level of virtual shells. Direct substitution into the corresponding Diophantine equation gives the exact coincidence of the calculated and actual mass of the Tau-lepton, which confirms the correctness of structural assumptions about the existence of a virtual shell of 82 particles.

b) The mass of the muon is known with high exactness, so its calculation will be even more convincing. Hypothetical reconstruction gives the following structural picture. Muon and Tau-lepton have only one identical structural component-it is the first shell (real electric charge and 11 virtual particles). Contribution of this component in the total mass is the same- $6.2521 \mathrm{~m}_{e}$. The third shell of 82 particles in the muon is simply absent. And the second shell is undergoing a very interesting change. The number of particles in this shell increases in such proportion that its contribution to the total mass of the muon increased by exactly 3 times, the threefold symmetry is realized not only at the level of quarks. This is achieved by increasing the number of particles from 20 particles in Tau-lepton structure up to 35 in muon structure. In a system of 21 particles (20 virtual + core) 210 pairwise interactions are possible combinatorial. But in a system of 36 particles (35 virtual + core) 630 pairwise interactions are possible combinatorial. Contribution of the second shell to the total muon mass in $m_{e}$ units is: $630 / \pi=200.5352$. Total mass of the muon is the sum of these two masses:

$$
6.2521 \cdot m_{e}+200.5352 \cdot m_{e}=206.7873 \cdot m_{e} .
$$

Actual mass of the muon is $206.7682 m_{e}$, the error of calculation less than $0.01 \%$. High accuracy of coincidence of the actual and estimated mass confirms the correctness of particles structural reconstruction.

In accordance with the empirical algorithm of increasing the number of particles in the shell from 20 up to 35 increases its contribution into the formed mass exactly three times. In contrast to this, for the initial shell of 19 or 21 particles the task of increasing three times has no solution in integers. This is a typical situation with Diophantus equations and convincing illustration of the fact that the reconstruction of the particle structure is not arbitrary, as it may seem at first glance

High accuracy of coincidence between calculated and actual mass of muon once more provides the validity of virtual shells model. But in the standard model all leptons are considered to be fundamental particles, i.e., not consisting of any structural components. Calculation of heavy leptons mass proves the component structure of these particles. Another thing is that the conceptual framework of the standard model does not assume the existence of virtual shells - this is a fundamentally new type of physical objects. There is concrete correlation between leptons virtual shells structure and their non-participation in strong nuclear interactions.

Heavy leptons have the unique structural feature. As we have seen both heavy leptons form virtual shell from 11 particles. For sure in details it is not a shell around real particle with electric charge, as in all other cases. From the symmetry it is practically obvious that heavy leptons form virtual shell with direct inclusion of real particle (real electric charge), using them as the twelfth vertex of the dodecahedron.

Among the elementary particles of different types, there is a phenomenon which in current terminology is called the existence of several "generations" of particles. There are similar particles with tremendous differences in mass values. For example, electron, muon and Tau-lepton represent three generations of leptons. Standard model has no explanation for this phenomenon. Now we see that the existence of several generations of the 
same particles is explained by the fact that the original particle can be supplemented with different variants of virtual shells. This leads to tremendous differences in the masses and is not accompanied by changes in any other properties of the particle.

General idea of the theory of quarks ("the principle of 1/3") in a latent form is realized in the field of gravity, in the formation of muon and Tau-lepton masses, i.e. even those particles, which electric charge which is formed without participation of quarks.

In search of the general harmony of nature, the parameters of planetary orbits many times, starting with I. Kepler, were compared with the configurations of regular polyhedra, the so-called "music of the spheres". In the fractal structure of virtual shells Tau-lepton really implemented configuration in the form of an icosahedron inside the dodecahedron. But such forms exist only at the stage of electromagnetic and gravitational interactions. At the stage of existence of the strong and weak nuclear forces, the geometric shape is changing dramatically. To a first approximation the shape undergoes a geometric inversion. But the specific reconstruction of the geometric shape of the particles on the stage with existence of nuclear forces remains a subject of future investigations.

\subsection{Pions Mass}

a) Let us try to predict the mass of such particles, which is the simplest complex of two quarks. Its shell should consists of 40 virtual particles,- -instead 60 particles in the shell of protons system with three quarks. Together with real core such particle is the complex of 41 particles with combinatorial possible 820 pairwise interactions. Their contribution to the total mass in $m_{e}$ units is $820 / \pi=261.01$. Contribution to the total mass of real core is $3 m_{e}$-two quarks and a single pairwise interaction (Figure 1(a)). Whole calculated mass is $264.01 m_{e}$.

And now let's check the prediction of the model on the facts. Particle with calculated mass really exist. It is the neutral pion, according to standard theory it is just the simplest complex of two quarks, more exactly-quark and antiquark. Actual mass $264.15 \mathrm{~m}_{e}$, calculation error is $0.04 \%$.

b) The empirical reconstruction of charged pion structure gives the following picture. Charged pion in comparison with neutral pion has 3 additional quarks forming electric charge. In total core has 5 quarks and 7 pairwise interactions combinatorial possible (Figure 1(b)). Contribution to the total mass of such core is $12 \mathrm{~m}_{e}$. Together with the shell mass $261.01 m_{e}$ whole calculated mass is $273.01 m_{e}$. Actual mass $273.12 m_{e}$, calculation error is $0.05 \%$. High accuracy of coincidence of the actual and estimated mass confirms the correctness of particles structural reconstruction.

\subsection{Mass of Neutral and Charged Vector Bosons, the Existence of Negative Masses}

Neutral and charged vector bosons are carriers of weak nuclear forces. More precisely, according to standard theory, photon and two types of vector bosons are carriers of the unified electroweak interactions. In comparison with other particles vector bosons have giant masses.

The actual mass of neutral vector boson ( $Z^{0}$ boson) is $(91,187 \pm 7) \mathrm{MeV}$, or $(178,450 \pm 15) m_{e}$, it's about 97.9 aum. Hypothetical reconstruction gives the following structural picture. The major part of this mass is formed by the virtual shell of 600 particles. Together with the core complex from particles 601 is obtained, where 180,300 pairwise interactions are possible combinatorial. The contribution of the shell to the total weight is $180,300 \mathrm{~m}_{e}-$ a little bit more than actual mass. It may seem strange, but the central core occurs to have negative mass. $\mathrm{Z}^{0}$ boson has the core which mass is modulo equal to the mass of the neutron:

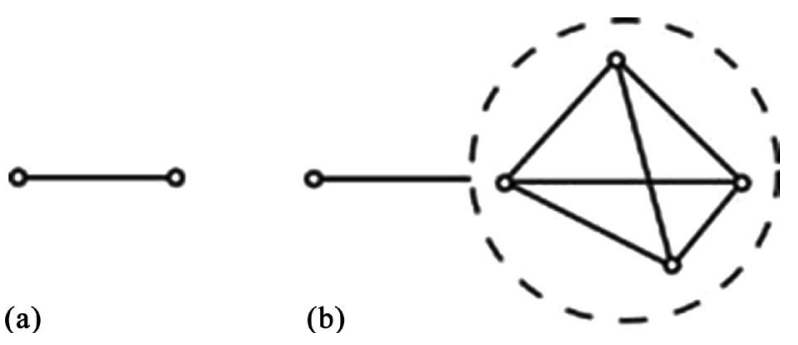

Figure 1. Structure of pion core: (a) Neutral pion, (b) Charged pion. 


$$
(178450 \pm 15) \cdot m_{e}-180300 \cdot m_{e}=(-1850 \pm 15) \cdot m_{e}
$$

This quantitative agreement cannot be accidental, and indirectly confirms the correctness of particles structural reconstruction.

The actual mass of the charged vector bosons $\left(\mathrm{W}^{+}, \mathrm{W}^{-}\right.$bosons) is $(80,150 \pm 100) \mathrm{MeV}$, or $(156,850 \pm 200) m_{e}$, it's about 86.0 aum. Hypothetical reconstruction gives the following structural picture. A virtual shell of such bosons contains 560 particles, i.e. differs from the shells of neutral $\mathrm{Z}^{0}$ boson to double of the "magic" number $(20+20)$ particles. The mass of a charged vector boson is formed as follows. In the complex of the real core and 560 virtual particles 157,080 pairwise interactions are possible combinatorial. The effect of the presence of the shell corresponds to $157,080 m_{e}$-a little bit more than actual mass. Charged bosons also have cores with negative mass:

$$
(156850 \pm 200) \cdot m_{e}-157080 \cdot m_{e}=(-230 \pm 200) \cdot m_{e} \cdot
$$

Probably, the core mass is modulo equal to muons mass. But for unique choice between muon and pion masses experimental measurements of the $\mathrm{W}^{+}, \mathrm{W}^{-}$bosons masses should be more exact.

Standard theory of the unified electroweak interactions (model by Salam and Weinberg) do not use the concept of negative mass, but the theory uses particles whose square seed mass are negative numbers. Accordingly such masses themselves are considered to be imaginary. Concepts of negative mass and imaginary mass have a lot in common and the differences are partly terminology. Using of negative mass concept is discussed below.

\section{Electromagnetic Constant 137.03605...}

It is logical to assume that the numerical value of the famous constant is also determined by quasicrystal vacuum. Hypothetical structural reconstruction involves that in addition to the gravitational shell around each elementary electric charge is formed electromagnetic shell of 60 virtual particles. In contrast with gravitation these particles are divided into two groups_- “electric” and "magnetic” types. Particles are divided into groups in the same proportion as electric charge between quarks-20 and 40 particles. Real electric charge in the center is included in both groups. So there are two groups of 21 and 41 particles. The cooperative interaction between groups is the result of $21 \times 41=861$ pairwise interactions. Using coefficient $2 \pi$ we obtain a dimensionless constant: $861 / 2 \pi=$ 137,032406 . Because of the participation of the central particle in two groups the amendment is necessary. A more exact value of the constant is given by the empirical ratio:

$$
\frac{861}{2 \pi}+\frac{\pi}{861}=137.036054774 \cdots
$$

Exactness of the coincidence of this number with the value recommended by the "Committee on Data for Science and Technology” (“CODATA”) is at the level of $0.0001 \%$ or 1 p.p.m. Actually in the experiments some metrological centers in different countries gets a value slightly more and some slightly less than the value calculated by me [12]. High accuracy of coincidence of the constants values confirms that the starting assumptions in the beginning of this section correspond to reality.

\section{Discussion}

\subsection{Dark Matter}

Dark matter is not the object simply invisible from the Earth, but extremely unusually effects of gravity itself. Direct astronomical observations allow us to set the parameters of the stellar orbits. And further attempts to calculate the mass of the Central body of the galaxy lead to a paradoxical picture. It turns out that the stars on the fringes of the galaxy rotate around the Central body of suitable mass, and the stars in the central part of the same galaxy revolve around the Central body whose mass is many times smaller. Such situation is impossible with a traditional point of view and absolutely natural in the scope of virtual shells model.

If we could look at the single proton using astronomical methods, the presence of a core and of a virtual shell, we would have interpreted as existence of protons own mass $6 m_{e}$ and the concomitant existence of dark matter with a mass of $1830 m_{e}$, i.e. 300 times greater. Dark matter is an aggregate of virtual shells of astronomical objects and with astronomical scales. Such shells really have properties of natural amplifiers of gravity, but the results can be observed only outside dimensions of the virtual shell. 
Perhaps our Sun has such virtual shell. Two American space vehicles "Pioneer 10" and "Pioneer 11" at intervals of a year in the same area on the periphery of the Solar system have experienced unexplained braking. I propose that vehicles crossed the suns virtual shell and got into the zone of action of solar gravity natural amplifier.

Only astronomers can professionally and critically compare the results of observation with hypothetical explanation in the framework of the virtual shells model. Just now it is only worth to say about the possibility of unexpected approach to the largest mass in the Universe.

\subsection{Higgs Bosons}

All 60 virtual particles in the shell of each proton are principally indistinguishable from each other. According to generally accepted particles classification they belong to the group of bosons. The particles in the composition of virtual shells of real elementary particles and atomic nuclei actually generate more than 99\% of ordinary matter mass. Namely, it is just the main property of the Higgs bosons. More detailed analysis of the properties of virtual particles from shells, as well as consideration of the complex of related issues is given in the books [5] [6].

It is crucial that the bosons in the virtual shells do not have any own masses. As we have seen along the whole Periodic table their contribution to real mass generation depends on the number of such virtual particles in concrete shell. That is why in standard theory mass of Higgs boson itself remained unknown. At the same time in the experiment it is impossible to separate bosons own mass and its contribution to the total mass of the real particle. In such situation the experimentally measured value must radically depend on what real particles were used as a target. A unique feature of the Higgs boson is that it creates a mass of other particles, but does not have any own mass. It seems to be obvious that only particle with such combination of properties by definition may be called the Creator of mass.

Information about experimental confirmation of the Higgs bosons existence in 2012 sparked a surge of scientific interest in this particle [13]. A review of the literature on this subject is not the subject of the article. But it is important to emphasize that the proposed model of discrete bosonic shells is not a marginal alternative to the Higgs bosons. Apparently, these are two different approaches to the same problem, which future research will merge into the overall picture.

Generation of real mass is a result of interaction between virtual shells particles and specific field. In absentia it was called the Higgs field. But I am sure that fundamentally it is just alternating gravitational field. About its properties we know very small, but enough to suggest that the most intriguing modern mysteries of biology and other sciences are directly connected with alternating gravitational fields. In such fields arise very considerable strengths, evident traces of their action are obviously seen, for example, on a usual school globe [6].

\subsection{Concept of Negative Mass}

Without any explanations about necessity of using the concept of negative mass long before me wrote the founder of solid state physics L. Brillouin [14]. Today it is worth to divide answers on two questions: why negative masses are necessary and why they are possible?

It is obvious that only particles with negative mass can generate attractive forces as result of exchanging interactions. The exchange of vector bosons leads to the appearance of attractive forces only because inside their structure exist components with negative mass, as we have seen above.

For elementary particles the concept of negative mass must be understood in a special sense. Existence of elementary particles not in empty space, but surrounded by physical vacuum inevitably changes the zero level of mass determining. In any real experiment it is impossible to provide selective force exerted on certain pre-selected elementary particle. Only force field in the surrounding space can be created.

The action of the gravitational field on elementary particle surrounded by vacuum conventionally can be compared to behavior in water steel balls and air bubbles. Actually air bubbles demonstrate only illusion of the negative mass, the air bubbles move against the forces of the gravitational field due to the force of Archimedes. But obvious understanding of the true situation is based on using concepts of density to water and air. To elementary particles and to physical vacuum mechanical concept of density is not applicable and adequate replacement does not exists.

In such a situation the zero level of mass determining is shifted and appears the illusion of negative mass. In 
vector bosons structure there are components whose interaction with the external gravitational field is weaker than that of the virtual vacuum particles. And just these structural components demonstrate the illusion of negative mass. This is a forced compromise due to lack of knowledge and imperfection of the conceptual apparatus. But compared with the representation of particles as mathematical points this seems a definite step forward.

\section{Conclusions}

The existence of natural standards of length and time defines the real distinguishing features of the microcosm. The standard time or frequency is created by the existence of quantum particles in the form of alternating two states with different properties, which creates the famous paradoxes - uncertainty principle, etc. Standard length is created by physical vacuum with special geometric structure similar to quasi-crystal.

In the world with such vacuum around elementary particles and atomic nuclei arise clearly structured shells, which form more than $99 \%$ of the mass of ordinary matter. Shells consist of bosons, which perhaps can be identified with the Higgs bosons.

The geometric structures of the virtual bosons shells are analogous to the fullerenes. Discrete variants of such structures create that set of masses of elementary particles and atomic nuclei that really exist in nature. In electromagnetism the virtual shell, geometrically similar to the fullerene $\mathrm{C}_{60}$, determines the numerical value of the famous constant $137.03605 \ldots$

\section{References}

[1] Golubev, S.N. (1981) Real Crystals in Coccolithophore Skeletons. Nauka, Moscow, 164 p.

[2] Golubev, S.N. (1987) Journal of General Biology, 6, 784-806.

[3] Golubev, S.N. (1996) Consciousness and Physical Reality, 1, 85-92.

[4] Golubev, S.N. (1996) Consciousness and Physical Reality, 1, 37-42.

[5] Golubev, S.N. (2013) Quasicrystalline Structure of the Vacuum: The Key to Unlocking the Secret of Living Cells and Quantum Particles. Librokom, Moscow, 256 p.

[6] Golubev, S.N. (2013) The Origin of Life and the Formation of Masses of Elementary Particles in the World of Quasicrystalline Vacuum. Folium, Moscow, 348 p.

[7] Golubev, S.N. (2014) Achievements in the Life Sciences, 8, 1-9. http://dx.doi.org/10.1016/j.als.2014.11.001

[8] Golubev, S.N. and Golubev, S.S. (2009) Look at the Physical Position of the Microcosm from the Biologist Perspective. Dalnauka, Vladivostok, Series of the "Achievements in the Life Sciences”, 245 p.

[9] Penrose R. (2003) Emperor’s New Mind: In Regard to Computers, Minds, and Laws of Physics. "Editorial URSS”, Moscow, $384 \mathrm{p}$.

[10] Schechtmann, D., Blech, S., Gratias, D. and Cahn, J.W. (1984) Physical Review Letters, 53, 1951-1953. http://dx.doi.org/10.1103/PhysRevLett.53.1951

[11] Kapitonov, I.M. (2002) Introduction to the Physics of Nuclei and Particles. “Editorial URSS”, Moscow, 384 p.

[12] Mohr, P.J. and Taylor, B.N. (2005) Reviews of Modern Physics, 77, 1-107. http://dx.doi.org/10.1103/RevModPhys.77.1

[13] Baggott, J. (2014) Higgs: The Inventation and Discovery. Centrpoligraph, Moscow, 255 p.

[14] Brillouin, L. (1972) A New Look at the Theory of Relativity. Mir, Moscow, 144 p. 